\title{
A Case Report of Alloimmune Hepatitis after Direct-acting Antiviral Treatment in a Liver Transplant Patient
}

\author{
Catherine Choi $^{1}$, Youssef Botros ${ }^{2}$, Jamil Shah ${ }^{3}$, Pei Xue ${ }^{4}$, Anja Jones ${ }^{5}$, Mark Galan ${ }^{5}$, \\ Raquel Olivo ${ }^{6}$, Mumtaz Niazi ${ }^{6}$, Flavio Paterno7 ${ }^{7}$ James Guarrera7 and Nikolaos T. Pyrsopoulos*6 \\ ${ }^{1}$ Department of Internal Medicine, Rutgers New Jersey Medical School, Newark, NJ, USA; ${ }^{2}$ Division of Gastroenterology, St. \\ Joseph's University Medical Center, Paterson, NJ, USA; ${ }^{3}$ Division of Gastroenterology \& Hepatology, The Brooklyn Hospital Center, \\ Brooklyn, NY, USA; ${ }^{4}$ Rutgers New Jersey Medical School, Newark, NJ, USA; ${ }^{5}$ Department of Pathology, Immunology \& Laboratory \\ Medicine, Rutgers New Jersey Medical School, Newark, NJ, USA; ${ }^{6}$ Division of Gastroenterology \& Hepatology, Department of \\ Medicine, Rutgers New Jersey Medical School, Newark, NJ, USA; ' Division of Liver Transplantation \& Hepatobiliary Surgery, \\ Department of Surgery, Rutgers New Jersey Medical School, Newark, NJ, USA
}

\begin{abstract}
Direct-acting antiviral (DAA) therapy is often well-tolerated, and adverse events from DAA therapy are uncommon. We report a case of a woman who underwent orthotopic liver transplant for chronic hepatitis $C$ infection and later developed alloimmune hepatitis shortly after starting DAA therapy for recurrent hepatitis $C$ infection. The patient developed acute alloimmune hepatitis approximately 2 weeks after starting treatment with sofosbuvir, velpatasvir, and voxilaprevir. This case report proposes a dysregulation of immune surveillance due to the DAA stimulation of host immunity and rapid elimination of hepatitis $C$ viral load as a precipitating factor for the alloimmune process, leading to alloimmune hepatitis in a post-transplant patient who starts on DAA.

Citation of this article: Choi $C$, Botros $Y$, Shah J, Xue $P$, Shah A, Galan $M$, et al. A case report of alloimmune hepatitis after direct-acting antiviral treatment in a liver transplant patient. J Clin Transl Hepatol 2020;8(4):459-462. doi: 10.14218/ JCTH.2020.00062.
\end{abstract}

\section{Introduction}

Previous studies have shown the link between hepatitis $C$ infection and autoimmune diseases ${ }^{1}$, and its presumed mechanism is an excessive immune response against hepatitis $C$ virus (HCV). Direct-acting antiviral (DAA) therapy is well tolerated in liver transplant patients, and serious adverse events from DAA are uncommon. De novo autoimmune hepatitis or alloimmune hepatitis are clinical entities that occur in patients who have undergone transplantation and are characterized by biochemical hepatitis, circulating autoantibodies, elevated IgG levels, and an inflammatory infiltration with interface hepatitis. ${ }^{2}$ Cases of alloimmune hepatitis induced after DAA

Keywords: Autoimmune hepatitis; Hepatitis C; Liver transplant.

Abbreviations: AIH, autoimmune hepatitis; DAA, direct-acting antiviral; $\mathrm{HCV}$, hepatitis $C$ virus; IgG, immunoglobulin; LT, liver transplantation; Tregs, regulatory T cells.

Received: 7 July 2020; Revised: 12 August 2020; Accepted: 24 August 2020

* Correspondence to: Nikolaos T. Pyrsopoulos, Division of Gastroenterology \& Hepatology, Department of Medicine, Rutgers New Jersey Medical School, Medical Science Building, Room I-506, 185 South Orange Avenue, Newark, NJ 07103, USA. Tel: +1-973-972-5252, E-mail: pyrsopni@njms.rutgers.edu in patients undergoing treatment for HCV infection have been reported in the literature. This is a case report of alloimmune hepatitis in a post-liver transplant patient shortly after starting DAA treatment for recurrent hepatitis $C$ in the graft.

\section{Case report}

The patient is a woman of Arabic background in her mid to late $50 \mathrm{~s}$ with a history of vitiligo and end-stage liver disease (induced by chronic HCV infection, genotype 4; treatmentexperienced), complicated with hepatocellular carcinoma, who presented to the emergency department with jaundice and elevated liver enzymes. She underwent liver transplantation (LT) 3 months prior.

The patient has a 14-year history of HCV infection and had been diagnosed with cirrhosis 3 years prior to the LT. She had received treatment for HCV 2 years prior to the LT with ombitasvir, paritaprevir, ritonavir, and ribavirin, but the treatment was discontinued after 2 months due to the development of hemolytic anemia. Unfortunately, she failed to achieve a sustained virologic response. She underwent orthotopic LT with a hepatitis B core antibody-positive and hepatitis $C$ antibody-positive graft. Prior to the current admission, the patient had been admitted to the hospital for investigation of elevated serum aminotransferase levels (aspartate aminotransferase of $227 \mathrm{U} / \mathrm{L}$, alanine aminotransferase of $219 \mathrm{U} / \mathrm{L}$ ) and HCV viral load of 734,703 IU/mL (Table 1). A liver biopsy showed portal inflammatory infiltrates, compatible with severe hepatitis, and features that were suggestive of recurrent HCV infection. DAA treatment was started with sofosbuvir, velpatasvir, and voxilaprevir. The patient responded to treatment with DAA, and the biochemical profile was significantly improved (Table 1 ).

Two weeks later, the patient complained of a 2-day experience of jaundice, nausea, vomiting, and diarrhea. The review of systems revealed subjective fevers, chills, and fatigue. She denied consumption of alcohol or use of other medications. Her vital signs were unremarkable. On examination, scleral icterus, jaundice, and moderate tenderness in the left upper quadrant and right upper quadrant, without guarding, were noted. The laboratory evaluation revealed a white blood cell count of 3,300 cells $/ \mathrm{mm}^{3}$ with a normal differential, aspartate aminotransferase of $1,395 \mathrm{U} / \mathrm{L}$, alanine aminotransferase of $1,359 \mathrm{U} / \mathrm{L}$, alkaline phosphatase of 
Table 1. Laboratory findings prior to and after DAA treatment

\begin{tabular}{llll}
\hline Lab finding & Prior to DAA treatment & At week 1 of DAA treatment & Post-DAA treatment \\
\hline Alkaline phosphatase, U/L & 84 & 579 & 79 \\
Aspartate aminotransferase, U/L & 227 & 1,201 & 24 \\
Alanine aminotransferase, U/L & 490 & 1,215 & 24 \\
Total bilirubin, $\mathrm{mg} / \mathrm{dL}$ & 0.3 & 11.5 & 0.5 \\
Direct bilirubin, $\mathrm{mg} / \mathrm{dL}$ & 0.1 & 537 & 0.2 \\
Hepatitis C viral load, IU/mL & 734,703 & Not detected & Not detected \\
Hepatitis B viral load, IU/mL & Not detected & 9 & Not detected \\
Tacrolimus level, $\mathrm{ng} / \mathrm{mL}$ & 8 & 7 & 7 \\
\hline
\end{tabular}

Elevated aminotransferase levels and other biochemical profiles improved after the treatment of DAA.

$601 \mathrm{U} / \mathrm{L}$, total bilirubin of $12.2 \mathrm{mg} / \mathrm{dL}$, direct bilirubin of $9.6 \mathrm{mg} / \mathrm{dL}$, and international normalized ratio of 1.5 . The tacrolimus level was $7 \mathrm{ng} / \mathrm{mL}$. Anti-nuclear antibody test was negative, but an elevated immunoglobulin $\mathrm{G}(\mathrm{IgG})$ level of $2,477 \mathrm{mg} / \mathrm{dL}$ was noted (Table 2). Other autoimmune serologies were unremarkable, including rheumatoid factor, anti-centromere antibody, anti-double stranded DNA, cyclic citrullinated peptide, liver-kidney microsomal antibody, Sjogren's antibodies SS-A and SS-B, anti-smooth muscle antibody, and anti-mitochondrial antibody.

A liver biopsy showed severe hepatitis with abundant plasma cells and interface activity, compatible with an alloimmune process (Figs. 1 and 2). C4D immunostaining was negative. The immunosuppressive and prophylactic therapies consisted of tacrolimus, mycophenolate, atovaquone, and entecavir. The patient was placed on $1 \mathrm{~g}$ of IV methylprednisolone and $\mathrm{N}$ acetyl cysteine infusion, which elicited subsequent clinical improvement. The patient was discharged on a steroid taper, in addition to her immunosuppression regimen. Hepatitis $B$ infection was ruled out as hepatitis B surface antigen, hepatitis B surface antibody, hepatitis B core antibody tests were all negative, and hepatitis B viral load was not detected by PCR. Human immunodeficiency virus screening test was also negative. Hepatitis E IgM antibody test was negative.

Table 2. Laboratory tests for the evaluation of alloimmune hepatitis

\begin{tabular}{|c|c|c|}
\hline Test & Normal range & Result \\
\hline Anti-nuclear antibody & No range & $\begin{array}{l}\text { Not } \\
\text { detected }\end{array}$ \\
\hline $\begin{array}{l}\text { Anti-mitochondrial } \\
\text { antibody }\end{array}$ & $\begin{array}{l}\text { Negative }<20 \\
U\end{array}$ & 5 \\
\hline $\begin{array}{l}\text { Anti-smooth muscle } \\
\text { antibody }\end{array}$ & $\begin{array}{l}\text { Negative }<20 \\
U\end{array}$ & 8.7 \\
\hline IgG & $\begin{array}{l}700-1,600 \\
\mathrm{mg} / \mathrm{dL}\end{array}$ & 2,477 \\
\hline Immunoglobulin M & $\begin{array}{l}40-230 \mathrm{mg} / \\
\mathrm{dL}\end{array}$ & 113 \\
\hline $\begin{array}{l}\text { Liver-kidney microsomal } \\
\text { antibody }\end{array}$ & $\begin{array}{l}\text { Negative }<20 \\
U\end{array}$ & $\begin{array}{l}\text { Not } \\
\text { detected }\end{array}$ \\
\hline
\end{tabular}

Serological testing for alloimmune hepatitis performed includes elevated IgG level, but anti-nuclear antibody, anti-mitochondrial antibody, and anti-smooth muscle antibody tests were negative. Of note, rheumatoid factor was negative initially but became positive approximately 16 months after the diagnosis of alloimmune hepatitis.
The patient ultimately completed a 12 -week course of DAA treatment. At her 3-month follow-up visit, the hepatic enzymes were found to be normalized by the maintenance steroid therapy with methylprednisolone $8 \mathrm{mg}$ PO daily. The patient was determined to have achieved a sustained virological response (Table 1). Repeat liver biopsy was conducted for elevated liver enzymes at 5 months after the initial liver biopsy (approximately 3 months after the completion of DAA treatment).The histopathologic findings of repeat liver biopsy again showed marked portal and lobular mixed inflammatory infiltrate comprised of lymphocytes, abundant plasma cells, and numerous eosinophils and neutrophils with interface activity, consistent with alloimmune hepatitis rather than acute cellular rejection. Hepatitis $C$ viral load was again undetectable. Of note, a repeat testing for rheumatoid factor was positive, approximately 16 months after the diagnosis of alloimmune hepatitis.

\section{Discussion}

Alloimmune hepatitis is a rare cause for graft dysfunction that can occur among patients who have undergone LT for reasons

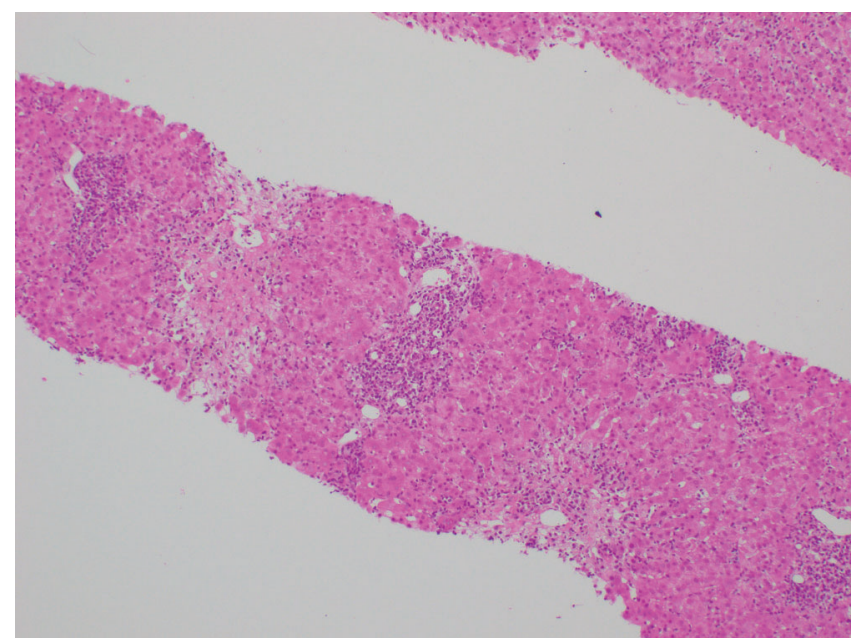

Fig. 1. Both portal and lobular regions demonstrate a mixed inflammatory infiltrate with abundant plasma cells.

Interface activity is present. The portal tract demonstrates extensive necrosis. 


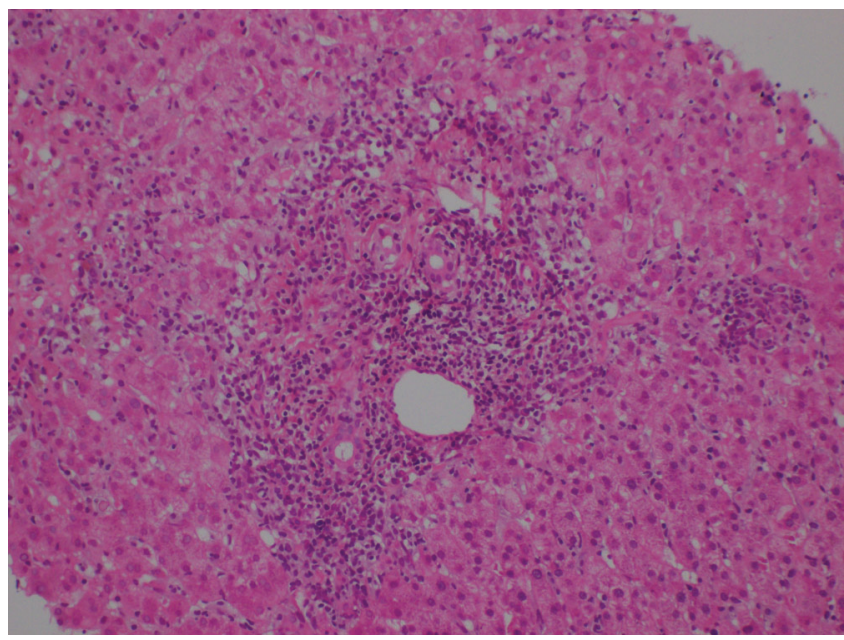

Fig. 2. High-power microscopy shows parenchymal collapse and necrosis. Plasmacytic infiltration is demonstrated.

other than autoimmune hepatitis (AIH). ${ }^{3-5}$ Several studies have demonstrated that DAA treatments are well tolerated in liver transplant patients and are successful at achieving sustained virologic response in more than $95 \%$ of patients. ${ }^{6}$ Serious adverse events can occur, however, including rashes, cytopenia, allograft rejection, severe anemia, and mortality, but these were only reported in $4 \%$ of cases $^{7}$ and thus our case is an unusual presentation following DAA therapy. The exact pathogenesis is not fully understood but believed to share similar histological and clinical findings with classical $\mathrm{AIH}^{3}$ Interferon-based antiviral treatments have been described to precipitate or exacerbate alloimmune hepatitis in the liver transplant population.8,9 Apart from its antiviral effects, interferon is a potent immunomodulator, and therefore it is not surprising that immune-mediated phenomena, including alloimmune hepatitis, is reported with interferonbased therapy of HCV infection. ${ }^{10} \mathrm{~A}$ possible mechanism for such immune-mediated complications is based on interferon therapy's effects on T-cell activation, which ultimately intensifies pro-inflammatory activity and enhances the presentation and release of antigens. ${ }^{10}$

We made our diagnosis of alloimmune hepatitis based on both histological and immunopathological findings from the liver biopsy, which showed marked portal and lobular mixed inflammatory infiltrate with interface activity. On the liver biopsy, C4D immunostaining was negative, and findings on repeat liver biopsy obtained 5 months after the completion of DAA were again consistent with alloimmune hepatitis rather than acute cellular rejection. We ruled out other causes that may mimic alloimmune hepatitis, such as rejection and viral hepatitis. The patient had a history of vitiligo, which is considered an autoimmune disease. Given the acute onset of the patient's presentation and the lack of evidence of graft rejection or co-infection on liver biopsy, the occurrence of alloimmune hepatitis was likely related to the immune system imbalance induced by a DAA regimen the occurrence of alloimmune hepatitis was likely related to the immune system imbalance induced by a DAA regimen. HCV viral load at the time of diagnosis of alloimmune hepatitis was $537 \mathrm{IU} / \mathrm{mL}$, and the mechanism appeared to be different from an HCV infectioninduced autoimmune reaction or drug-induced liver injury.
Repeat testing for hepatitis $\mathrm{B}$ and $\mathrm{C}$ viral loads were all negative. The ImmuKnow ${ }^{\mathrm{TM}}$ assay is an immune monitoring test, and the higher test value indicates a greater risk of rejection. ${ }^{11}$ In our patient, the ImmuKnow ${ }^{\mathrm{TM}}$ assay result was negative.

As the anti-HCV activity of DAA therapy has the potential to stimulate host immunity, activation of the immune system in the setting of rapid elimination of HCV might favor a dysregulation of immune surveillance. ${ }^{12-14}$ Although the exact pathogenesis of classic AIHs is not clear, one possible etiology is an imbalance between regulatory T cells and proinflammatory cells. ${ }^{15-18}$ Regulatory T cells (Tregs) are a subpopulation of T cells that have been demonstrated to suppress pathology in multiple autoimmune diseases. Reduction in frequency and function of Tregs has been reported in peripheral blood in patients with AIH in several studies. ${ }^{16,17}$ On the contrary, there is a parallel increase in Treg frequency in the inflamed liver tissue ${ }^{19}$ but a decline in the functional capacity of the Tregs in AIH. ${ }^{21}$ The functional capacity of Tregs in the liver is essential to control ongoing hepatitis by suppressing the effector cells in the inflamed liver in $\mathrm{AIH} .^{21}$

Previous studies showed that IgG4 can down-modulate the immune system, and IgG4-rich infiltrates were more elevated in allograft recipients with de novo AIH compared with $\mathrm{AIH}$ in native liver or kidney allograft recipients with plasma-cell-rich rejection. ${ }^{22}$ In our patient, serological IgG $(2,447 \mathrm{mg} / \mathrm{dL})$ was elevated but an IgG4 level was not tested. With a better understanding of the underlying mechanism behind Treg activity in alloimmune hepatitis, further advancements in targeting the Tregs may become alternative treatments to immunosuppressive therapies for treating alloimmune hepatitis. In summary, we report a case of alloimmune hepatitis in a liver transplant patient with recurrent HCV responding to DAA therapy. A thorough follow up of patients undergoing DAA therapy after LT for alloimmune hepatitis might be warranted.

\section{Funding}

None to declare.

\section{Conflict of interest}

Dr. Pyrsopoulos is a recipient of research grants from Bayer, Beigene, Intercept, Mallinckrodt, Eisai, Novartis, Resusix, Saro, Valeant, Gilead, Genfit, Grifols, Prometheus, and Zydus. Dr. Pyrsopoulos has also served as a consultant for Bayer and Eisai. Dr. Guarrera is a recipient of research grants from and has served a consultant for Organ Recovery Systems Inc for work in Organ Preservation. The other authors have no conflict of interests related to this publication.

\section{Author contributions}

Review of the literature ( $C C, Y B, J S, P X)$, drafting of the manuscript (CC, YB, PX), review of the manuscript (RO, MN, $\mathrm{FP}, J G$ ), providing figures and descriptions (AJ, MG), critical revision of the manuscript (NTP).

\section{References}

[1] De Maria C, Ghidotti I, Grillo F, Giannini EG. Successful DAA Treatment and Global Improvement in a Cirrhotic Patient with Concomitant HCV Infection and Autoimmune Hepatitis. Dig Dis Sci 2019;64(2):591-593. 
[2] Dooley J, Pinzani M, Lok ASF, Garcia-Tsao G. Sherlock's diseases of the liver and biliary system. Thirteenth edition. ed.

[3] Guido M, Burra P. De novo autoimmune hepatitis after liver transplantation. Semin Liver Dis 2011;31(1):71-81.

[4] Liberal R, Longhi MS, Grant CR, Mieli-Vergani G, Vergani D. Autoimmune hepatitis after liver transplantation. Clin Gastroenterol Hepatol 2012;10(4): 346-353.

[5] Neil DA, Hubscher SG. Current views on rejection pathology in liver transplantation. Transpl Int. 2010;23(10):971-983.

[6] Hull MW, Yoshida EM, Montaner JS. Update on Current Evidence for Hepatitis C Therapeutic Options in HCV Mono-infected Patients. Curr Infect Dis Rep 2016;18(7):22.

[7] Liu J, Ma B, Cao W, et al. Direct-acting antiviral agents for liver transplant recipients with recurrent genotype 1 hepatitis $C$ virus infection: Systematic review and meta-analysis. Transpl Infect Dis 2019;21(2):e13047.

[8] Kerkar N, Dugan C, Rumbo $C_{\text {, }}$ et al. Rapamycin successfully treats posttransplant autoimmune hepatitis. Am J Transplant. 2005;5(5):1085-1089.

[9] Kontorinis N, Agarwal K, Elhajj N, Fiel MI, Schiano TD. Pegylated interferoninduced immune-mediated hepatitis post-liver transplantation. Liver Transp 2006;12(5):827-830.

[10] Selzner N, Guindi M, Renner EL, Berenguer M. Immune-mediated complications of the graft in interferon-treated hepatitis $C$ positive liver transplant recipients. J Hepatol 2011;55(1):207-217.

[11] Crespo-Leiro MG, Barge-Calballero E, Paniagua-Martin MJ, Barge-Calballero G, Suarez-Fuentetaja N. Update on Immune Monitoring in Heart Transplatation. Current Transplantation Reports 2015;2:329-337.

[12] Debes JD, van Tilborg M, Groothuismink ZMA, et al. Levels of Cytokines in Serum Associate With Development of Hepatocellular Carcinoma in Patients With HCV Infection Treated With Direct-Acting Antivirals. Gastroenterology 2018;154(3):515-517 e513.

\section{Choi C. et al: Alloimmune hepatitis following DAA treatment}

[13] Grandhe S, Frenette CT. Occurrence and Recurrence of Hepatocellular Carcinoma After Successful Direct-Acting Antiviral Therapy for Patients With Chronic Hepatitis C Virus Infection. Gastroenterol Hepatol (N Y) 2017;13 (7):421-425.

[14] Tatsumi T, Takehara T. Impact of natural killer cells on chronic hepatitis $C$ and hepatocellular carcinoma. Hepatol Res 2016;46(5):416-422.

[15] Liberal R, Grant CR, Longhi MS, Mieli-Vergani G, Vergani D. Regulatory T cells: Mechanisms of suppression and impairment in autoimmune liver disease. IUBMB Life 2015;67(2):88-97.

[16] Longhi MS, Hussain MJ, Mitry RR, et al. Functional study of CD4+CD25+ regulatory $T$ cells in health and autoimmune hepatitis. J Immunol 2006; 176(7):4484-4491.

[17] Longhi MS, Ma Y, Bogdanos DP, Cheeseman P, Mieli-Vergani G, Vergani D. Impairment of $\mathrm{CD} 4(+) \mathrm{CD} 25(+)$ regulatory T-cells in autoimmune live disease. J Hepatol 2004;41(1):31-37.

[18] Longhi MS, Ma Y, Mitry RR, et al. Effect of CD4+ CD25+ regulatory T-cells on CD8 T-cell function in patients with autoimmune hepatitis. $J$ Autoimmun 2005;25(1):63-71.

[19] Oo YH, Weston CJ, Lalor PF, et al. Distinct roles for CCR4 and CXCR3 in the recruitment and positioning of regulatory $T$ cells in the inflamed human liver. J Immunol 2010;184(6):2886-2898.

[20] Chen YY, Jeffery HC, Hunter S, et al. Human intrahepatic regulatory T cells are functional, require IL-2 from effector cells for survival, and are susceptible to Fas ligand-mediated apoptosis. Hepatology 2016;64(1):138-150.

[21] Than NN, Jeffery HC, Oo YH. Autoimmune Hepatitis: Progress from Global Immunosuppression to Personalised Regulatory T Cell Therapy. Can J Gastroenterol Hepatol 2016;2016:7181685

[22] Castillo-Rama M, Sebagh M, Sasatomi E, et al. "Plasma cell hepatitis" in liver allografts: identification and characterization of an IgG4-rich cohort. Am J Transplant 2013;13(11):2966-2977. 